\title{
Diffusion Capacity of the Lung for Carbon Monoxide/Alveolar Volume Ratio
}

National Cancer Institute

\section{Source}

National Cancer Institute. Diffusion Capacity of the Lung for Carbon Monoxide/Alveolar

Volume Ratio. NCI Thesaurus. Code C120830.

The calculated ratio of the lung's alveolar volume with respect to its diffusion capacity for carbon monoxide. 